\title{
The ARGO-YBJ legacy to next generation wide field-of-view experiments
}

\author{
Giuseppe Di Sciascio ${ }^{\text {a }}$ \\ INFN - Rome Tor Vergata, Italy
}

\begin{abstract}
The ARGO-YBJ experiment has been in stable data taking for more than 5 years at the YangBaJing Cosmic Ray Observatory (Tibet, P.R. China, $4300 \mathrm{~m}$ a.s.1., $606 \mathrm{~g} / \mathrm{cm}^{2}$ ). With a duty-cycle greater than $86 \%$ the detector collected about $5 \times 10^{11}$ events in a wide energy range, from few hundreds $\mathrm{GeV}$ up to about $10 \mathrm{PeV}$. High altitude location and detector features make ARGO-YBJ capable of investigating a wide range of important issues in Cosmic Ray and Astroparticle Physics by imaging the front of atmospheric showers with unprecedented resolution and detail. In this contribution some of the latest physics results obtained by ARGO-YBJ in gamma-ray astronomy and in cosmic ray physics are summarized. The prospects of $\mathrm{TeV}$ gamma-ray observations with new ground-based wide field-of-view detectors are presented.
\end{abstract}

\section{Introduction}

The riddle of the origin of Cosmic Rays (hereafter CR) is unsolved since more than one century. The identification of the galactic sources able to accelerate particles beyond $\mathrm{PeV}\left(=10^{15} \mathrm{eV}\right)$ energies, the so-called 'PeVatrons', is certainly one of the main open problems of high energy astrophysics. In fact, even there is no doubt that galactic CR are accelerated in SuperNova Remnants (SNRs), the capability of SNRs to accelerate CRs up to the 'knee' of the all-particle energy spectrum $\left(\sim 3 \times 10^{15} \mathrm{eV}\right)$ and above is still under debate. The determination of the maximum energy at which protons are accelerated inside their sources (the 'proton knee'), as well as the measurement of the evolution of the heavier component across the knee, are the key for understanding acceleration mechanisms and the propagation processes in the Galaxy, and to investigate the transition from Galactic to extra-galactic CRs.

The study of CRs is based on two complementary approaches:

1. Measurement of energy spectrum, elemental composition and anisotropy in the CR arrival direction distribution, the three basic observables crucial for understanding origin, acceleration and propagation of the radiation.

2. Search of their sources through the observation of neutral radiation (photons and neutrinos), which points back to the emitting sources not being affected by the magnetic fields.

The integrated study of charged CRs and of gamma rays/neutrinos (the 'cosmic ray connection'), which should trace high energy hadronic interactions mainly nearby the still unidentified acceleration sites, is one of the most important (and exciting) fields in the so-called 'multimessenger astronomy'.

\footnotetext{
a e-mail: disciascio@roma2.infn.it
}

In the PeV energy region an accurate measurement of the CR primary spectrum can be carried out only by ground-based Extensive Air Showers (hereafter EAS) arrays, detectors capable of simultaneously and continuously viewing all the overhead sky due to an effective field-of-view of about $2 \mathrm{sr}$ and a duty-cycle of $\sim 100 \%$. These two characteristics make them the key instruments for studying the highest energy gamma rays, extended sources and transient phenomena. The all-sky survey provides un unbiased map of the sky useful to enable the detection of unexpected sources, to provide testing ground for new theoretical ideas and to provide targets for in-depth observations by satellites or Cherenkov telescopes (IACTs).

The ARGO-YBJ experiment has been proposed to investigate the 'cosmic ray connection' through a combined study of photon- and charged particle-induced EAS with the same detector. The combination of the high elevation of the site and the high granularity of the readout provides very important advantages in various aspects of CR physics. In fact, in addition to the decrease of the threshold energy down to few hundreds $\mathrm{GeV}$, the location of the experiment above $4000 \mathrm{~m}$ a.s.l. ensures that shower fluctuations are small (we are working in the shower maximum region) and that all nuclei produce showers with nearly the same electromagnetic size. The low energy threshold is crucial to evaluate the absolute energy scale of the detector exploiting the Moon shadow technique and for the overposition with direct measurements carried out by balloon/satellite-born detectors allowing the cross-calibration between different energy scales. The independence of the size on the mass of the primary particle determines the size - energy relation to be better defined, allowing the study of the elemental composition around the knee in a very efficient and reliable way.

In this paper the latest results obtained by ARGO-YBJ in measuring the $\mathrm{p}+\mathrm{He}$ energy spectrum, the CR anisotropy and the diffuse gamma-ray emission from the Galactic

(c) The Authors, published by EDP Sciences. This is an Open Access article distributed under the terms of the Creative Commons Attribution License 4.0 (http://creativecommons.org/licenses/by/4.0/). 
Plane are summarized. We note that these topics can be studied only by EAS-arrays, and not by IACTs, due to their capability in studying the extended gamma emissions and in selecting showers induced by different nuclei. We also introduce two new projects for future wide field-ofview experiments in the Northern and in the Southern hemispheres.

\section{The ARGO-YBJ experiment}

ARGO-YBJ was a full coverage air shower detector constituted by a central carpet $\sim 74 \times 78 \mathrm{~m}^{2}$, made of a single layer of Resistive Plate Chambers (RPCs) with $\sim 93 \%$ of active area, enclosed by a guard ring partially instrumented $(\sim 20 \%)$ up to $\sim 100 \times 110 \mathrm{~m}^{2}$. The apparatus has a modular structure, the basic data acquisition element being a cluster $\left(5.7 \times 7.6 \mathrm{~m}^{2}\right)$, made of $12 \mathrm{RPCs}$ $\left(2.85 \times 1.23 \mathrm{~m}^{2}\right.$ each $)$. Each chamber is read by 80 external strips of $6.75 \times 61.80 \mathrm{~cm}^{2}$ (the spatial pixels), logically organized in 10 independent pads of $55.6 \times 61.8 \mathrm{~cm}^{2}$ which represent the time pixels of the detector [1]. The readout of 18,360 pads and 146,880 strips is the experimental output of the detector. In addition, in order to extend the dynamical range up to $\mathrm{PeV}$ energies, each chamber is equipped with two large size pads $\left(139 \times 123 \mathrm{~cm}^{2}\right)$ to collect the total charge developed by the particles hitting the detector [2]. The RPCs are operated in streamer mode by using a gas mixture (Ar 15\%, Isobutane 10\%, TetraFluoroEthane $75 \%$ ) for high altitude operation [3]. The high voltage settled at $7.2 \mathrm{kV}$ ensures an overall efficiency of about $96 \%$ [4]. The central carpet contains 130 clusters and the full detector is composed of 153 clusters for a total active surface of $\sim 6,700 \mathrm{~m}^{2}$. The total instrumented area is $\sim 11,000 \mathrm{~m}^{2}$. For each event the location and timing of every detected particle is recorded, allowing the reconstruction of the lateral distribution and the arrival direction. The trigger is based on a time correlation among the pad signals depending on their relative distance. In this way, all the shower events giving a number of fired pads $\mathrm{N}_{\text {pad }} \geq \mathrm{N}_{\text {trig }}$ in the central carpet in a time window of 420 ns generate the trigger. The whole system has been in stable data taking from November 2007 to January 2013, with the trigger condition $\mathrm{N}_{\text {trig }}=20$ and a duty cycle $\geq 86 \%$. The trigger rate is $\sim 3.5 \mathrm{kHz}$ with a dead time of $4 \%$.

Because of the small pixel size, the detector is able to record events with a particle density exceeding 0.003 particles $\mathrm{m}^{-2}$, keeping good linearity up to a core density of about 15 particles $\mathrm{m}^{-2}$. This high granularity allows a complete and detailed threedimensional reconstruction of the front of air showers at an energy threshold of a few hundreds GeV. Showers induced by high energy primaries $(>100 \mathrm{TeV})$ are also imaged by the charge readout of the large size pads which allows to study the structure of the shower core region up to particle densities of $\sim 10^{4} / \mathrm{m}^{2}$ [2].

Details on the analysis procedure (e.g., reconstruction algorithms, data selection, background evaluation, systematic errors) are discussed in [5-7]. The performance of the detector (angular resolution, pointing accuracy, energy scale calibration) and the operation stability are continuously monitored by observing the Moon shadow, i.e., the deficit of CRs detected in its direction $[6,8]$. The measured angular resolution is better than $0.5^{\circ}$ for
CR-induced showers with energy $\mathrm{E}>5 \mathrm{TeV}$ and the overall absolute pointing accuracy is $\sim 0.1^{\circ}$. According to MonteCarlo simulations the angular resolution for $\gamma$-induced events results smaller by $30 \%-40 \%$. The absolute rigidity scale uncertainty of ARGO-YBJ is estimated at $10 \%$ level in the range $1-30 \mathrm{TeV} / \mathrm{Z}[6,8]$.

\subsection{Measurement of the Cosmic Ray light component $(p+\mathrm{He})$ energy spectrum}

A measurement of the CR primary $\mathrm{p}+\mathrm{He}$ energy spectrum has been carried out with ARGO-YBJ from few TeV up to about $5 \mathrm{PeV}$ exploiting different approaches:

- 'Digital-Bayes' analysis, based on the strip multiplicity, i.e. the picture of the EAS provided by the strip/pad system, in the few $\mathrm{TeV}-300 \mathrm{TeV}$ energy range. The selection of light elements (i.e. $\mathrm{p}+\mathrm{He}$ ) is based on the particle lateral distribution. The energy is reconstructed, on a statistical basis, by using a Bayesian approach $[9,10]$.

- 'Analog-Bayes' analysis, based on the RPC charge readout [2], covers the $30 \mathrm{TeV}-5 \mathrm{PeV}$ energy range. The energy is reconstructed (as in the previous analysis), on a statistical basis, by using a Bayesian approach.

- 'Hybrid measurement', carried out by ARGOYBJ and a wide field-of-view Cherenkov telescope (WFCTA), in the $100 \mathrm{TeV}-3 \mathrm{PeV}$ region. The selection of $(\mathrm{p}+\mathrm{He})$-originated showers is based on the shape of the Cherenkov image and on the particle density in the core region measured by ARGO-YBJ $[11,12]$.

We note that with ARGO-YBJ the selection of $(p+H e)-$ originated showers is performed not by means of an unfolding procedure after the measurement of electronic and muonic sizes, but on an event-by-event basis exploiting showers topology, i.e. the lateral distribution of charged secondary particles, in the shower core region. This approach is made possible by the full coverage of the central carpet, the high segmentation of the read-out and the high altitude location of the experiment that retains the characteristics of showers lateral distribution in the core region.

The energy spectrum of the $\mathrm{p}+\mathrm{He}$ component measured by ARGO-YBJ with different analyses is shown in the Fig. 1. The p+He measured by CREAM [13] is also shown for comparison. The average spectrum can be described by a single power-law with a spectral index of $-2.63 \pm 0.06$ up to about $600 \mathrm{TeV}$. The absolute flux at $400 \mathrm{TeV}$ is $(1.79 \pm 0.16) \times 10^{-11} \mathrm{GeV}^{-1} \mathrm{~m}^{-2} \mathrm{sr}^{-1} \mathrm{~s}^{-1}$. This result is consistent for what concerns the spectral index and absolute flux with the measurements carried out by CREAM. The flux difference is about $10 \%$ and can be explained with a difference in the experiments energy scale less than $\pm 4 \%$ [11]. This result is very important to fix the energy scale of the experiment. Below $10 \mathrm{TeV}$ the absolute energy scale of ARGO-YBJ is calibrated at $10 \%$ level exploting the displacement of the Moon shadow under the effect of the Geomagnetic Field. Above this energy the overposition with CREAM allows to check the energy scale at a few percent level up to the $100 \mathrm{TeV}$ range. 


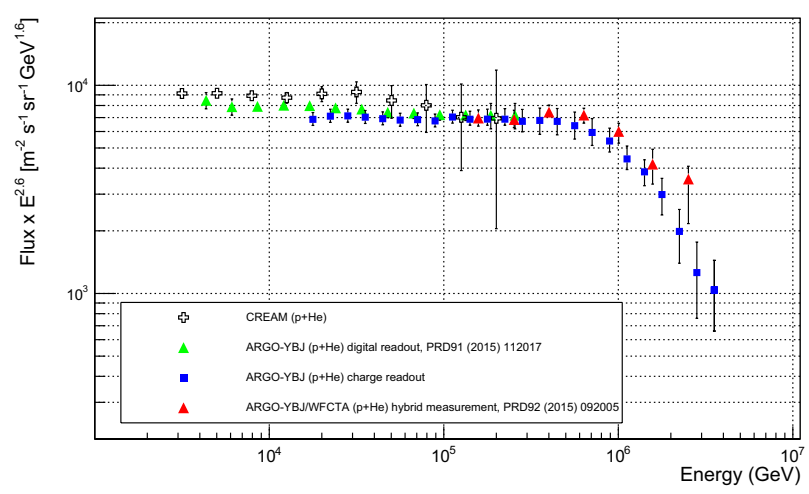

Figure 1. Light ( $\mathrm{p}+\mathrm{He}$ ) component energy spectrum of primary CRs measured by ARGO-YBJ. The $(\mathrm{p}+\mathrm{He})$ energy spectrum measured by CREAM [13] is shown for comparison. The systematic uncertainty is shown by the error bars.
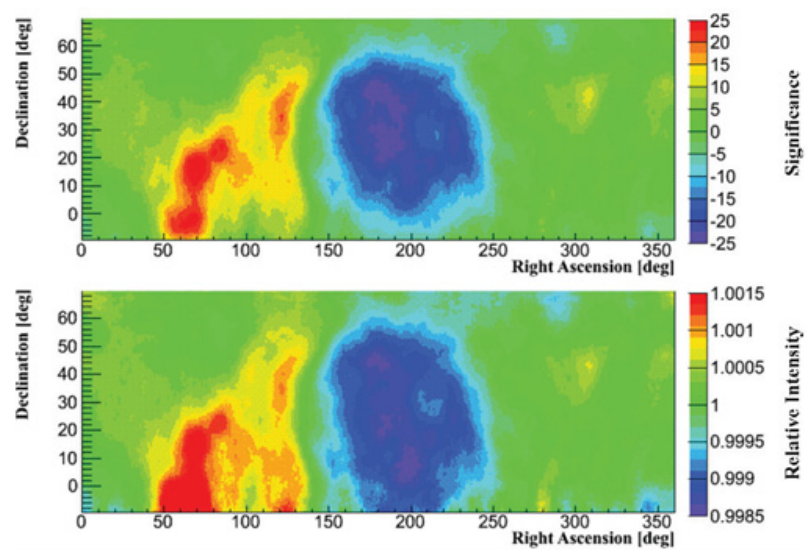

Figure 2. Large scale anisotropy observed by ARGO-YBJ [17]. The upper panel shows the significance map in standard deviations (s.d.), the lower panel gives the CR relative intensity. The sky maps are in the equatorial coordinate system. The corresponding proton median energy is about $1 \mathrm{TeV}$.

For higher energies the different analyses show clear evidence of a knee-like structure in the $\mathrm{p}+\mathrm{He}$ spectrum starting from about $700 \mathrm{TeV}$. The energy spectra measured by ARGO-YBJ/WFCTA can be fitted with a broken power-law function with $\mathrm{E}_{k}=700 \pm 230 \mathrm{TeV}, \mathrm{J}\left(\mathrm{E}_{k}\right)=$ $(4.65 \pm 0.27) \times 10^{-12} \mathrm{GeV}^{-1} \mathrm{~m}^{-2} \mathrm{~s}^{-1} \mathrm{sr}^{-1}, \beta_{1}=-2.56 \pm$ 0.05 and $\beta_{2}=-3.24 \pm 0.36$ above the knee [12].

ARGO-YBJ confirms the observation of the knee in the all-particle energy spectrum at few $\mathrm{PeV}$, but the clear observation of a knee in the $\mathrm{p}+\mathrm{He}$ energy spectrum below the PeV suggests that the elemental composition in the few $\mathrm{PeV}$ range is heavier than Helium, at variance with the current interpretation of the electron and muon KASCADE data, which attributes the knee of the all-particle spectrum to the proton component [14-16].

These results demonstrate the possibility of exploring the $\mathrm{CR}$ properties in a wide energy range with a single ground-based detector without exploiting the measurement of the muon size, thus reducing the uncertainties due to hadronic interaction models at a few percent level.

\subsection{Cosmic ray anisotropy}

The "Large Scale Anisotropy (LSA) observed by ARGOYBJ at about $1 \mathrm{TeV}$ in 2008 and 2009, during the latest minimum of the solar activity, is shown in Fig. 2 [17].
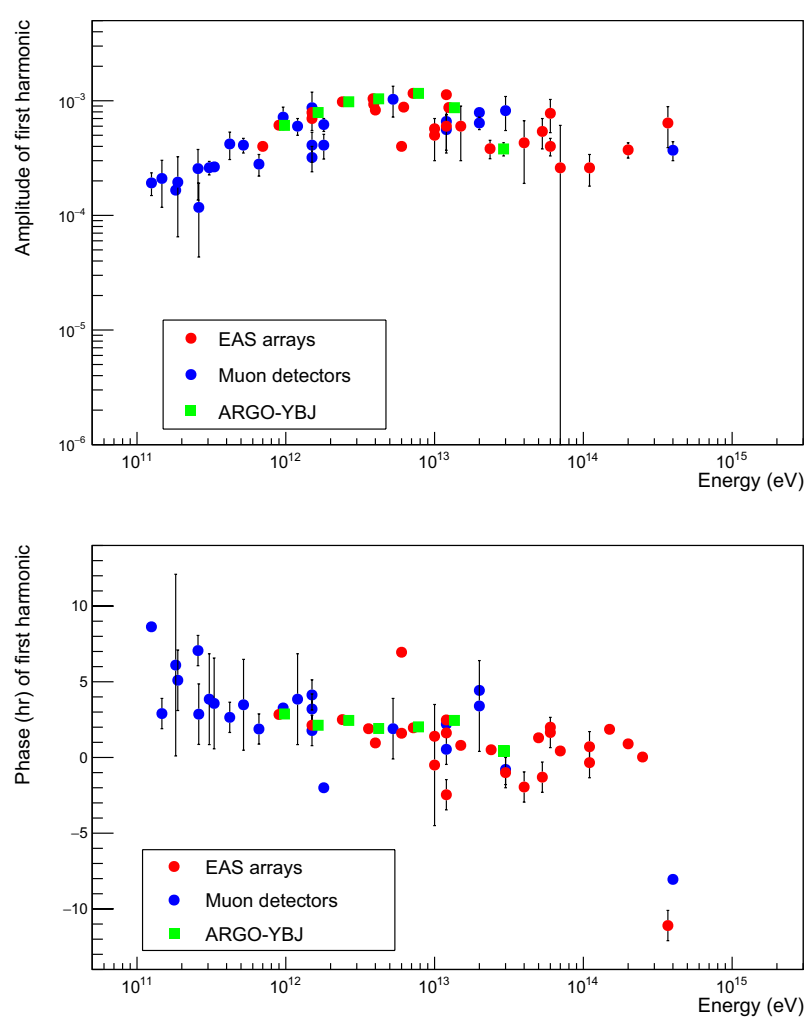

Figure 3. Amplitude and phase of the first harmonic (upper and lower plots, respectively) measured by ARGO-YBJ [17] compared with a compilation of data obtained by different experiments (muon telescopes or EAS-arrays) as a function of the CR primary energy (for details and references see [18]).

The existence of two distinct broad anisotropy regions in sidereal time, one showing an excess of CRs (called "tailin"), distributed around $40^{\circ}$ to $90^{\circ}$ in Right Ascension (R.A.), the other a deficit (the "loss cone"), distributed around $150^{\circ}$ to $240^{\circ}$ in R.A., has been clearly observed by ARGO-YBJ. The center of the "tail-in" component is close to the direction of the heliospheric tail, which is opposite to the proper motion direction of the solar system. The center of the "loss cone" deficit component points to the direction of the north Galactic pole. These observations rule out the hypothesis that a ComptonGetting effect due to the motion of the heliosphere with respect to the local insterstellar medium (expected as a dipole with a maximum in the direction of the Galactic Center decl. $\simeq 49^{\circ}$, R.A. $\simeq 315^{\circ}$ and a larger amplitude $\left.3.5 \times 10^{-3}\right)$ is a major source of the anisotropy [18, 19].

In Fig. 3 the amplitude and phase of the first harmonic (upper and lower plots, respectively) measured by different experiments are shown as a function of the primary CR energy. As can be seen from the plots

a) The amplitude of the CR anisotropy is extremely small $\left(10^{-4}-10^{-3}\right)$.

b) A slow increase of the amplitude to a maximum at a few $\mathrm{TeV}$ is observed. After the maximum the anisotropy decreases to a minimum at $\sim 100 \mathrm{TeV}$. Evidence for a new increase for higher energies appears from data.

c) The phase of the first harmonic is nearly constant (slowly decreasing) around $0 \mathrm{hrs}$. A dramatic change of phase is observed around $\sim 100 \mathrm{TeV}$, suggesting a 


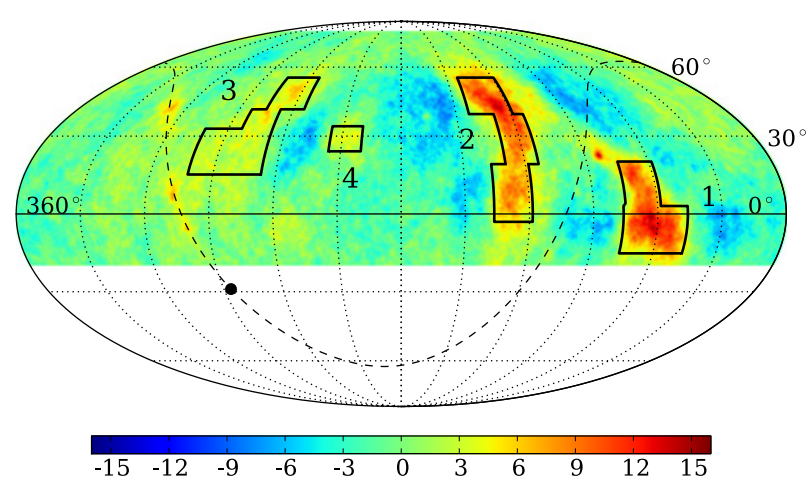

Figure 4. ARGO-YBJ sky-map in equatorial coordinates [20]. The color scale shows the statistical significance of the observation in s.d.. The dashed line represents the Galactic Plane and the black point the Galactic Center.

dipole opposite to the initial one. This observation clearly rules out that the Compton-Getting effect is a major source of the anisotropy.

d) The results obtained by ARGO-YBJ (green squares) are in fair agreements with other experiments.

With ARGO-YBJ we collected such large statistics to allow the investigation of anisotropic structures on smaller angular scale than the ones corresponding to the dipole and the quadrupole ("Medium/Small Scale Anisotropy”, MSA), showing that the CR intensity has quite a complicated structure unaccountable simply by kinetic models. Figure 4 shows the ARGO-YBJ sky map in equatorial coordinates as obtained with about $3.7 \cdot 10^{11}$ events reconstructed with a zenith angle $\leq 50^{\circ}$ (selecting the declination region $\delta \sim-20^{\circ} \div 80^{\circ}$ ) [20]. According to the simulation, the median energy of the isotropic CR proton flux is $\mathrm{E}_{p}^{50} \approx 1.8 \mathrm{TeV}$ (mode energy $\approx 0.7 \mathrm{TeV}$ ). The boxes represent the parametrization of the 4 regions of interest selecting the part of signal more than 3 s.d. [20].

The most evident features are observed by ARGOYBJ around the positions $\alpha \sim 120^{\circ}, \delta \sim 40^{\circ}$ and $\alpha \sim 60^{\circ}$, $\delta \sim-5^{\circ}$. These regions are observed with a statistical significance of about 15 s.d. . On the left side of the sky map, several new extended features are visible, though less intense than the ones aforementioned. The area $195^{\circ} \leq$ $R . A . \leq 290^{\circ}$ seems to be full of a few-degree excesses not compatible with random fluctuations (the statistical significance is up to 7 s.d.). We note that the region 4 is located in the "loss cone" of the LSA, near the North Galactic pole. The observation of regions 3 and 4 is reported by ARGO-YBJ for the first time. We note that the regions over which ARGO-YBJ observes significant MSA have total extension $\sim 0.8$ sr, i.e one third of the ARGO-YBJ field of view in celestial coordinates.

\subsection{Diffuse gamma-ray emission from the galactic plane}

Diffuse gamma rays are produced by relativistic electrons by bremsstrahlung or inverse Compton scattering on background radiation fields, or by protons and nuclei via the decay of $\pi^{0}$ produced in hadronic interactions with interstellar gas. Thus, the space distribution of this emission can trace the location of the CR sources and the distribution of interstellar gas. On the other hand, the diffuse emission provides a background in the search for point sources.

In the $\mathrm{GeV}$ energy range, the EGRET data showed a significant excess compared to expectations (the 'EGRET GeV excess') [22] likely due to instrumental effects [23] because not confirmed by the more recent and accurate Fermi-LAT data $[24,25]$. A diffuse gamma ray flux at TeV energies from the $30^{\circ}<l<110^{\circ}$ longitudinal range of the Galactic plane has been reported by Milagro [26-28].

The measured flux, once connected to the EGRET data by a power law with differential spectral index of -2.61 , reveals a "TeV excess" in the diffuse gamma ray spectrum, being this flux 5-10 times higher than expectations [29]. However, as will be discussed in the following, the Milagro result does not take into account the contributions from the Cygnus Cocoon and from the overlapping point or extended sources TeV J2032+4130, VER J2019+407 and VER J2016+372 [30].

The events collected by ARGO-YBJ have been analysed to determine the diffuse gamma ray emission in the Galactic plane at Galactic longitudes $25^{\circ}<l<100^{\circ}$ and Galactic latitudes $|b|<5^{\circ}$ [21]. This analysis was carried out in the energy range from $\sim 350 \mathrm{GeV}$ to $\sim 2 \mathrm{TeV}$, connecting the region explored by Fermi/LAT with that investigated by Milagro. In particular, the analysis was focused on two selected regions of the Galactic plane, i.e., $40^{\circ}<l<100^{\circ}$ and $65^{\circ}<l<85^{\circ}$ (the Cygnus region), where Milagro observed an excess with respect to that predicted by current models.

In the Galaxy region $40^{\circ}<l<100^{\circ},|b|<5^{\circ}$, after masking the discrete sources and subtracting the residual contribution, an excess with a statistical significance of 6.1 s.d. above the background is found. The spectral analysis provides the three fluxes shown in Fig. 5, upper plot, at median energies $350 \mathrm{GeV}, 680 \mathrm{GeV}$ and $1.47 \mathrm{TeV}$ (with uncertainties of about $30 \%$ ). The fit to the ARGO-YBJ data with a power law gives a spectral index $-2.90 \pm 0.31$, and the corresponding flux at $1 \mathrm{TeV}$ is compatible with the extrapolation of the Fermi/LAT template [31].

On the other hand, as mentioned, the first measurement of the diffuse $\mathrm{TeV}$ (integral) flux from the Galactic plane made by Milagro [32] revealed a "TeV excess" in the diffuse gamma ray spectrum with respect to expectations [33]. This Milagro measurement, converted into differential flux, is only $34 \%$ greater than the value expected from the extrapolation of the Fermi/LAT template, and within the experimental uncertainties (see triangle with error bars in Fig. 5, upper plot). Moreover, considering that the Milagro result does not take into account the contributions from the Cygnus Cocoon (not yet discovered at the time of the measurement) [30] and from overlapping point and extended sources, the discrepancy with the Fermi/LAT predictions is almost cancelled out. Therefore, the full set of measurements with ground-based detectors is in agreement with direct observations by Fermi/LAT, and the evidence of any "TeV excess", requiring additional sources or particle production processes other than those producing Galactic cosmic rays, is ruled out.

In the Galactic region $65^{\circ}<l<85^{\circ},|b|<5^{\circ}$, after masking the discrete sources and the Cygnus Cocoon and subtracting the residual contribution, an excess of 4.1 s.d. is left. This direction points into our spiral arm at the remarkable Cygnus star-forming region, located at a 

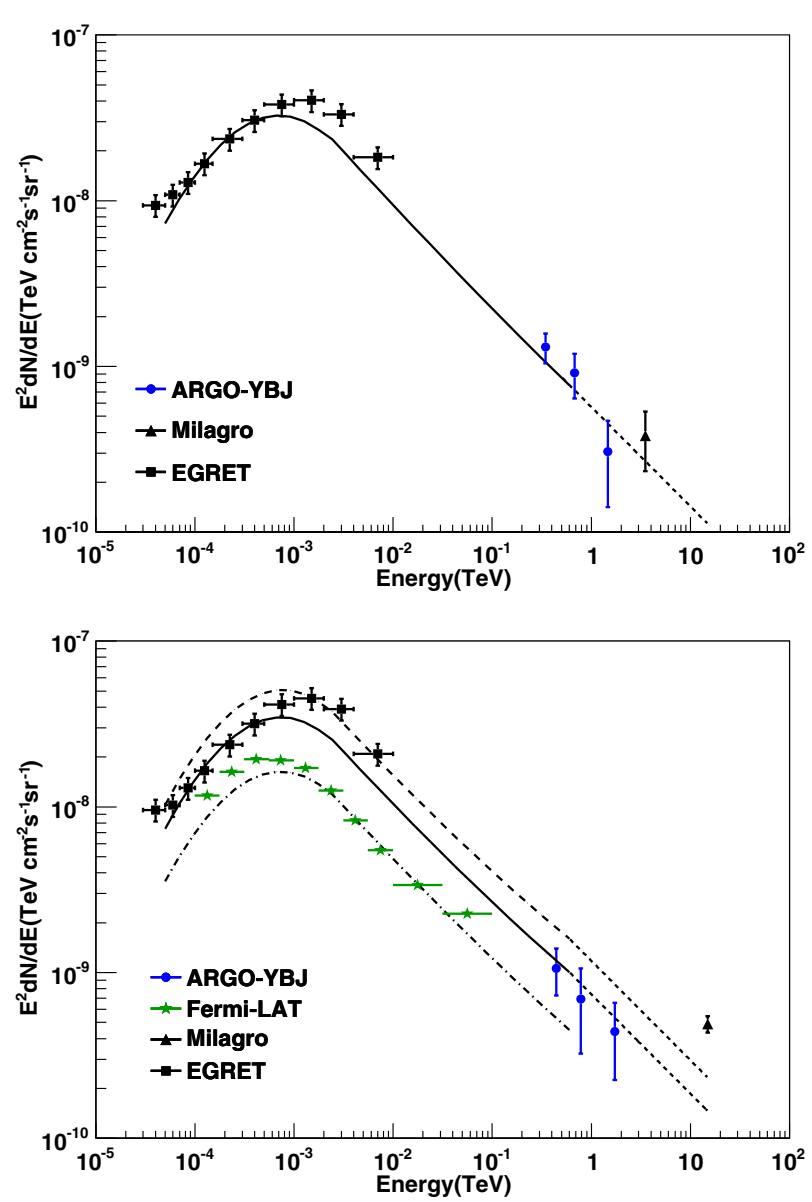

Figure 5. Upper plot: energy spectrum of the diffuse gamma ray emission measured in the Galactic region $40^{\circ}<l<100^{\circ}$, $|b|<5^{\circ}$ [21]. Lower plot: energy spectra of the diffuse gamma ray emission measured by ARGO-YBJ and EGRET in the Galactic region $65^{\circ}<l<85^{\circ},|b|<5^{\circ}$, by Milagro in the region $65^{\circ}<l<85^{\circ},|b|<2^{\circ}$ and by Fermi/LAT in $72^{\circ}<l<88^{\circ}$, $|b|<15^{\circ}$ [21]. The lines indicate the energy spectra expected from the Fermi/LAT template (with spectral index -2.6 , which also rules its short-dashed extension) in the different sky regions investigated by the detectors [31].

distance of about $1.4 \mathrm{kpc}$. The spectral energy distribution of gamma ray emission is shown in Fig. 5, lower plot (filled stars) [21] together with the spectrum expected from the Fermi/LAT template (dot-dashed line) [31]. Milagro measured the diffuse gamma ray emission from the region $65^{\circ}<l<85^{\circ},|b|<2^{\circ}$ at a median energy of $15 \mathrm{TeV}$ [28], obtaining the flux reported as a filled triangle in Fig. 5, lower plot. For comparison, the longdashed line shows the expected energy spectrum for this region according to the Fermi/LAT template. The Milagro flux results about $75 \%$ higher than the Fermi template, suggesting the presence of an excess. The spectral analysis of ARGO-YBJ data provides the three fluxes shown in Fig. 5 lower plot at median energies $440 \mathrm{GeV}, 780 \mathrm{GeV}$ and $1.73 \mathrm{TeV}$ (with uncertainties of about $40 \%$ ). The fit to ARGO-YBJ data with a power law gives a spectral index $-2.65 \pm 0.44$, and the corresponding flux at $1 \mathrm{TeV}$ is about $10 \%$ lower than the extrapolation of the Fermi/LAT template. These data do not show any excess at energies around $1 \mathrm{TeV}$ which corresponds to the excess found by Milagro at a median energy of $15 \mathrm{TeV}$. Again, this
Table 1. The spectral indices of gamma diffuse emission measured by ARGO-YBJ in different regions of the Galactic plane for $|b|<5^{\circ}$.

\begin{tabular}{|c|c|c|}
\hline Locations & Statistical Significance & Spectral Index \\
\hline $25^{\circ}<l<100^{\circ}$ & 6.9 s.d. & $-2.80 \pm 0.26$ \\
\hline $40^{\circ}<l<100^{\circ}$ & 6.1 s.d. & $-2.90 \pm 0.31$ \\
\hline $65^{\circ}<l<85^{\circ}$ & 4.1 s.d. & $-2.65 \pm 0.44$ \\
\hline $25^{\circ}<l<65^{\circ} \&$ & 5.6 s.d. & $-2.89 \pm 0.33$ \\
$85^{\circ}<l<100^{\circ}$ & & \\
\hline \hline
\end{tabular}

discrepancy can be explained taking into account that the contribution of all the discrete gamma ray sources was not completely removed from the Milagro data. According to the ARGO-YBJ data, the $1 \mathrm{TeV}$ flux associated to the Cygnus Cocoon is of the same order of the diffuse flux [30].

The spectral indices measured by ARGO-YBJ in different regions of the Galactic plane are reported in Table 1 [21]. The statistical significance of the observations in standard deviations (s.d.) is shown in the second column. The TeV flux averaged over the Cygnus region $65^{\circ}<l<$ $85^{\circ}$ cover the energy range $400 \mathrm{GeV}-2 \mathrm{TeV}$ and follow a power law with spectral index $-2.65 \pm 0.44$, a value very close to that found for $\mathrm{TeV}$ emission from the Cygnus Cocoon and for the $\mathrm{p}+\mathrm{He}$ component measured by ARGOYBJ, indicating the presence of young CRs accelerated by a nearby source [30].

\section{What's Next?}

\subsection{The LHAASO experiment}

A new project, developed starting from the experience of the high altitude experiment ARGO-YBJ, strategically built to study with unprecedented sensitivity the energy spectrum, the elemental composition and the anisotropy of CRs in the energy range between $10^{12}$ and $10^{17} \mathrm{eV}$, as well as to act simultaneously as a wide aperture ( $\sim 2 \mathrm{sr})$, continuosly-operated gamma-ray telescope in the energy range between $10^{11}$ and $10^{15} \mathrm{eV}$ is the LHAASO experiment $[34,35]$. The remarkable sensitivity of LHAASO in CR physics and gamma astronomy would play a key-role in the comprehensive general program to explore the "High Energy Universe".

The first phase of LHAASO will consist of the following major components (see Fig. 6):

- $1 \mathrm{~km}^{2}$ array (LHAASO-KM2A) for electromagnetic particle detectors (ED) divided into two parts: a central part including 4931 scintillator detectors $1 \mathrm{~m}^{2}$ each in size ( $15 \mathrm{~m}$ spacing) to cover a circular area with a radius of $575 \mathrm{~m}$ and an outer guard-ring instrumented with 311 EDs (30 m spacing) up to a radius of $635 \mathrm{~m}$.

- An overlapping $1 \mathrm{~km}^{2}$ array of 1146 underground water Cherenkov tanks $36 \mathrm{~m}^{2}$ each in size, with $30 \mathrm{~m}$ spacing, for muon detection (MD, total sensitive area $\sim 42,000 \mathrm{~m}^{2}$ ).

- A close-packed, surface water Cherenkov detector facility with a total area of about $78,000 \mathrm{~m}^{2}$ (LHAASO-WCDA).

- 18 wide field-of-view air Cherenkov telescopes (LHAASO-WFCTA). 


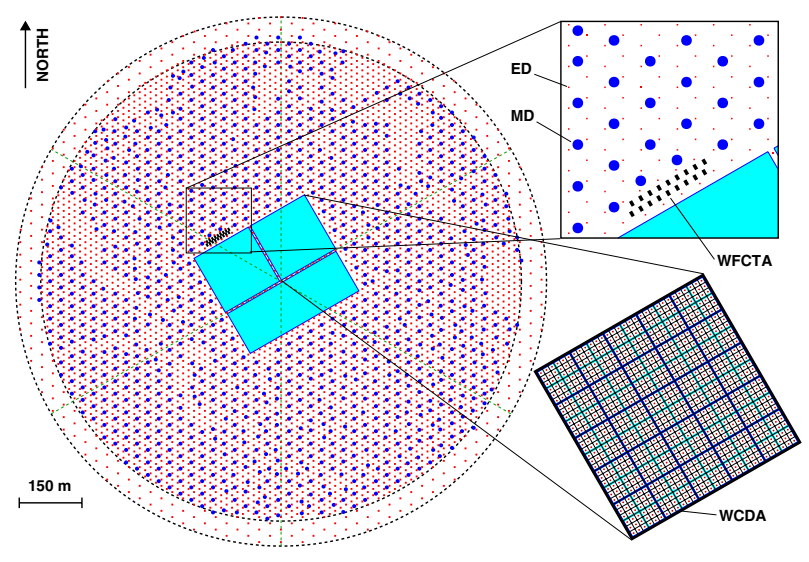

Figure 6. Layout of the LHAASO experiment. The insets show the details of one pond of the WCDA and of the KM2A array constituted by two overimposed arrays of electromagnetic particle detectors (ED) and of muon detectors (MD). The telescopes of the WFCTA, located at the edge of a pond, are also shown.

LHAASO is under installation at high altitude (4410 $\mathrm{m}$ asl, $\left.600 \mathrm{~g} / \mathrm{cm}^{2}, 29^{\circ} 21^{\prime} 31^{\prime \prime} \mathrm{N}, 100^{\circ} 08^{\prime} 15^{\prime \prime} \mathrm{E}\right)$ in the Daochen site, Sichuan province, P.R. China. The commissioning of one fourth of the detector will be implemented in 2018 . The completion of the installation is expected by the end of 2021.

In Table 2 the characteristics of the LHAASO-KM2A array are compared with other experiments. As can be seen, LHAASO will operate with a coverage of $\sim 0.5 \%$ over a $1 \mathrm{~km}^{2}$ area. The sensitive area of muon detectors is unprecedented and about 17 times larger than CASA-MIA, with a coverage of about $5 \%$ over $1 \mathrm{~km}^{2}$.

LHAASO will enable studies in CR physics and gamma-ray astronomy that are unattainable with the current suite of instruments:

1) LHAASO will perform an unbiased sky survey of the Northern sky with a detection threshold of a few percent $\mathrm{Crab}$ units at sub- $\mathrm{TeV} / \mathrm{TeV}$ energies and around $100 \mathrm{TeV}$ in one year (Fig. 7). This sensitivity grants a high discovery potential of flat spectrum Geminga-like sources not observed at $\mathrm{GeV}$ energies. This unique detector will be capable of continuously surveying the $\gamma$-ray sky for steady and transient sources from about $100 \mathrm{GeV}$ to $1 \mathrm{PeV}$.

From its location LHAASO will observe at TeV energies and with high sensitivity about 30 of the sources catalogued by Fermi-LAT at lower energy, monitoring the variability of 15 AGNs (mainly blazars) at least.

2) The sub-TeV/TeV LHAASO sensitivity will allow to observe AGN flares that are unobservable by other instruments, including the so-called $\mathrm{TeV}$ orphan flares.

3) LHAASO will study in detail the high energy tail of the spectra of most of the $\gamma$-ray sources observed at $\mathrm{TeV}$ energies, opening for the first time the $100-1000 \mathrm{TeV}$ range to the direct observations of the high energy cosmic ray sources. LHAASO's wide field-of-view provides a unique discovery potential.

4) LHAASO will map the Galactic diffuse gammaray emission above few hundreds $\mathrm{GeV}$ and thereby measure the CR flux and spectrum throughout the Galaxy with high sensitivity. The measurement of the space distribution of diffuse $\gamma$-rays will allow to trace the location of the $\mathrm{CR}$ sources and the distribution of interstellar gas.

5) The high background rejection capability in the 10-100 TeV range will allow LHAASO to measure the isotropic diffuse flux of ultrahigh energy $\gamma \mathrm{ra}$ diation expected from a variety of sources including Dark Matter and the interaction of $10^{20} \mathrm{eV}$ CRs with the $2.7 \mathrm{~K}$ microwave background radiation. In addition, LHAASO will be able to achieve a limit below the level of the IceCube diffuse neutrino flux at $10-100 \mathrm{TeV}$, thus constraining the origin of the IceCube astrophysical neutrinos.

6) LHAASO will allow the reconstruction of the energy spectra of different CR mass groups in the $10^{12}-10^{17} \mathrm{eV}$ with unprecedented statistics and resolution, thus tracing the light and heavy components through the knee of the all-particle spectrum.

7) LHAASO will allow the measurement, for the first time, of the CR anisotropy across the knee separately for light and heavy primary masses.

8) The different observables (electronic, muonic and Cherenkov components) that will be measured in LHAASO will allow a detailed investigation of the role of the hadronic interaction models, therefore investigating if the EAS development is correctly described by the current simulation codes.

9) LHAASO will look for signatures of WIMPs as candidate particles for DM with high sensitivity for particles masses above $10 \mathrm{TeV}$. Moreover, axion-like particle searches are planned, where conversion of gamma-rays to/from axion-like particles can create distinctive features in the spectra of gamma-ray sources and/or increase transparency of the universe by reducing the Extragalactic Background Light (EBL) absorption. Testing of Lorentz invariance violation as well as the search for Primordial Black Holes and Q-balls will also be part of the scientific programme of the experiment.

In the next decade CTA-North and LHAASO are expected to be the most sensitive instruments to study Gamma-Ray Astronomy in the Northern hemisphere from about $20 \mathrm{GeV}$ up to PeV.

\subsection{The LATTES project}

To maximize the scientific return for Galactic sources, a future wide field-of-view $\gamma$-ray experiment should be located at sufficiently Southern latitude to continuously monitor the Galactic Center and the Inner Galaxy. To lower the energy threshold down to hundred $\mathrm{GeV}$ range such an instrument would require extreme altitude location ( $>5000 \mathrm{~m}$ asl), high secondary photons conversion capability and a full coverage approach (with a coverage factor $>90 \%$ ) with high segmentation of the readout.

The LATTES project will consist of a carpet of RPCs on top of Water Cherenkov Detectors (WCD) of small dimensions [36].

The basic element, shown in Fig. 8, is composed by one WCD, with a rectangular horizontal surface of $3 \mathrm{~m} \times 1.5 \mathrm{~m}$ 
Table 2. Characteristics of different EAS-arrays.

\begin{tabular}{|c|c|c|c|c|}
\hline Experiment & Altitude (m) & $\begin{array}{l}\text { e.m. Sensitive Area } \\
\left(\mathrm{m}^{2}\right)\end{array}$ & $\begin{array}{l}\text { Instrumented Area } \\
\left(\mathrm{m}^{2}\right)\end{array}$ & Coverage \\
\hline LHAASO & 4410 & $5.2 \times 10^{3}$ & $1.3 \times 10^{6}$ & $4 \times 10^{-3}$ \\
\hline TIBET AS $\gamma$ & 4300 & 380 & $3.7 \times 10^{4}$ & $10^{-2}$ \\
\hline IceTop & 2835 & $4.2 \times 10^{2}$ & $10^{6}$ & $4 \times 10^{-4}$ \\
\hline ARGO-YBJ & 4300 & 6700 & 11,000 & 0.93 (central carpet) \\
\hline KASCADE & 110 & $5 \times 10^{2}$ & $4 \times 10^{4}$ & $1.2 \times 10^{-2}$ \\
\hline KASCADE-Grande & 110 & 370 & $5 \times 10^{5}$ & $7 \times 10^{-4}$ \\
\hline CASA-MIA & 1450 & $1.6 \times 10^{3}$ & $2.3 \times 10^{5}$ & $7 \times 10^{-3}$ \\
\hline & & $\begin{array}{c}\mu \text { Sensitive Area } \\
\left(\mathrm{m}^{2}\right)\end{array}$ & $\begin{array}{c}\text { Instrumented Area } \\
\left(\mathrm{m}^{2}\right)\end{array}$ & Coverage \\
\hline LHAASO & 4410 & $4.2 \times 10^{4}$ & $10^{6}$ & $4.4 \times 10^{-2}$ \\
\hline TIBET AS $\gamma$ & 4300 & $4.5 \times 10^{3}$ & $3.7 \times 10^{4}$ & $1.2 \times 10^{-1}$ \\
\hline KASCADE & 110 & $6 \times 10^{2}$ & $4 \times 10^{4}$ & $1.5 \times 10^{-2}$ \\
\hline CASA-MIA & 1450 & $2.5 \times 10^{3}$ & $2.3 \times 10^{5}$ & $1.1 \times 10^{-2}$ \\
\hline
\end{tabular}

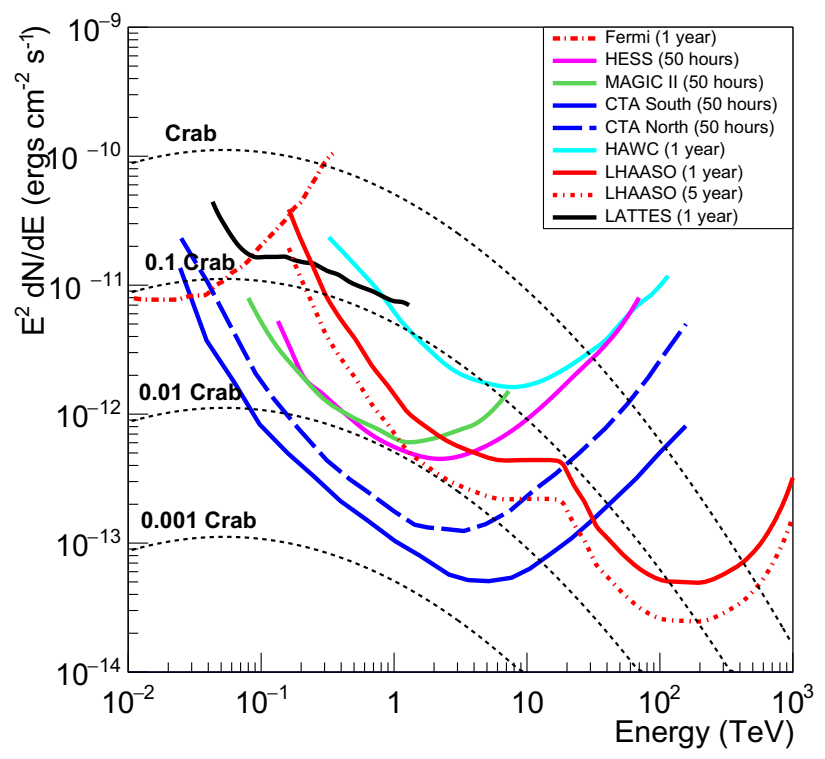

Figure 7. Differential sensitivity of LHAASO to a Crablike point gamma-ray source compared to other experiments (multiplied by $\mathrm{E}^{2}$ ). The Crab Nebula spectrum, extrapolated to $1 \mathrm{PeV}$, is reported as a reference together with the spectra corresponding to $10 \%, 1 \%$ and $0.1 \%$ of the Crab flux.

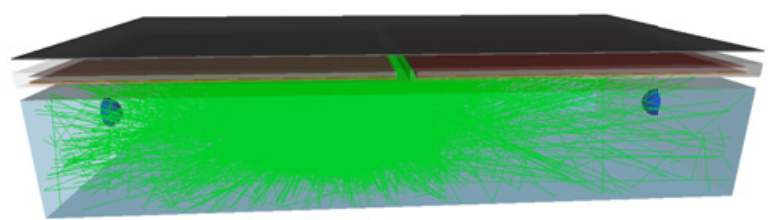

Figure 8. Basic LATTES detector station, with one WCD covered with RPCs and a thin slab of lead [36]. The green lines show the tracks of the Cherenkov photons inside the water.

and a depth of $0.5 \mathrm{~m}$, covered by two RPCs, each with a surface of $(1.5 \times 1.5) \mathrm{m}^{2}$, with a lead layer on top $(5.6 \mathrm{~mm})$ to exploit the secondary photons conversion. The WCD read-out will be provided by two $15 \mathrm{~cm}$ PMTs at both ends of the smallest vertical face of the tank. The read-out of the RPCs will be provided by 16 charge collecting pads. This hybrid detector is expected to improve the trigger selection at low energies and the rejection of the background of charged nuclei. The shower energy will be reconstructed from the total signal, defined as the sum of the number of photoelectrons in all WCD stations. The proposed experiment will consist of an array of $60 \times 30$ stations, covering an effective area of about $10,000 \mathrm{~m}^{2}$ located at $5200 \mathrm{~m}$ asl. The different detectors will be separated by a small distance (roughly $0.5 \mathrm{~m}$ ) to allow access to PMTs and RPCs. An angular resolution better than $2^{\circ}$ is expected in the $100 \mathrm{GeV}$ range.

The 1-year expected sensitivity, at level of $15 \%$ of the Crab Nebula flux in the $100-400 \mathrm{GeV}$ energy range, is shown in Fig. 7.

\section{Conclusions}

The ARGO-YBJ detector exploiting the full coverage approach and the high segmentation of the readout imaged the front of atmospheric showers with unprecedented resolution and detail. The physics of Galactic CRs has been studied with a combined measurement of photon- and charged particle-induced showers.

With ARGO-YBJ we demonstrated that RPCs can be safely operated at extreme altitudes for many years. The benefits in the use of RPCs in ARGO-YBJ were:

1. high efficiency detection of low energy showers (energy threshold $\sim 300 \mathrm{GeV}$ ) by means of the dense sampling of the central carpet;

2. unprecedented wide energy range investigated by means of the digital/charge read-outs $(\sim 300 \mathrm{GeV} \rightarrow$ $10 \mathrm{PeV}$ );

3. good angular resolution and unprecedented details in the core region by means of the high granularity of the read-outs.

Open problems in cosmic ray physics push the construction of a new generation of EAS arrays to study, in the $10^{11}-10^{18} \mathrm{eV}$ energy range, photon- and charged-induced events at the same time.

LHAASO is the most ambitious project for a new generation multi-component wide field-of-view experiment in the Northern hemisphere.

A new EAS array to study the $100 \mathrm{GeV} \gamma$-sky with high sensitivity and to monitor the Galactic Center should 
be a high priority. The LATTES project is a hybrid detector to exploit both the ARGO-YBJ full coverage approach with RPCs and the HAWC/LHAASO technique to reject the background of charged cosmic rays with a water Cherenkov detector. Such an experiment, if located in the Southern hemisphere at $5200 \mathrm{~m}$ asl with an instrumented area of $10,000 \mathrm{~m}^{2}$, can reach a sensitivity of order of $10 \%$ of the Crab Nebula flux in one year in the $100 \mathrm{GeV}$ energy range thus monitoring the Galactic Center and complementing the coming Cherenkov Telescope Array.

\section{References}

[1] G. Aielli et al., NIM A562, 92 (2006)

[2] B. Bartoli et al., Astropart. Phys. 67, 47 (2015)

[3] C. Bacci et al., NIM A443, 342 (2000)

[4] G. Aielli et al., NIM A608, 246 (2009)

[5] G. Aielli et al., Astrophys. J. Lett. 714, L208 (2010)

[6] B. Bartoli et al., Phys. Rev. D 84, 022003 (2011)

[7] B. Bartoli et al., Astrophys. J. 734, 110 (2011)

[8] B. Bartoli et al., Phys. Rev. D 85, 022002 (2012)

[9] B. Bartoli et al., Phys. Rev. D 85, 092005 (2012)

[10] B. Bartoli et al., Phys. Rev. D 91, 112017 (2015)

[11] B. Bartoli et al., Chinese Phys. C 38, 045001 (2014)

[12] B. Bartoli et al., Phys. Rev. D 92, 092005 (2015)

[13] Y.S. Yoon et al., Astrophys. J. 728, 122 (2011)

[14] T. Antoni et al., Astropart. Phys. 24, 1 (2005)

[15] W.D. Apel et al., Astropart. Phys. 31, 86 (2009)

[16] W.D. Apel et al., Astropart. Phys. 47, 54 (2013)

[17] B. Bartoli et al., Astrophys. J. 809, 90 (2015)
[18] G. Di Sciascio and R. Iuppa, On the observation of the Cosmic Ray Anisotropy below $10^{15} \mathrm{eV}$, in "Homage to the Discovery of Cosmic Rays, the Meson-Muons and Solar Cosmic Rays", Chapter 9, pagg. 221-257 (Nova Science Publishers, Inc., New York, 2013). Preprint: arXiv: 1407.2144

[19] G. Di Sciascio, ASTRA Proc. 2, 27 (2015)

[20] B. Bartoli et al., Phys. Rev. D 88, 082001 (2013)

[21] B. Bartoli et al., Astrophys. J. 806, 20 (2015)

[22] S.D. Hunter et al., Astrophys. J. 481, 205 (1997)

[23] F.W. Stecker et al., Astropart. Phys. 29, 25 (2008)

[24] A.A. Abdo et al., Phys. Rev. Lett. 103, 251101 (2009)

[25] A.A. Abdo et al., Astrophys. J. 710, 133 (2010)

[26] R. Atkins et al., Phys. Rev. Lett. 95, 251103 (2005)

[27] A. Abdo et al., Astrophys. J. 658, L33 (2007)

[28] A.A. Abdo et al., Astrophys. J. 688, 1078 (2008)

[29] F. Aharonian et al., Rep. Prog. Phys. 71, 096901 (2008)

[30] B. Bartoli et al., Astrophys. J. 790, 152 (2014)

[31] M. Ackermann et al., Astrophys. J. Suppl. 209, 34 (2013)

[32] R. Atkins et al. Phys. Rev. Lett. 95, 251103 (2005)

[33] T. Prodanović, B. D. Fields \& J. F. Beacom, Astropart. Phys. 27, 10 (2007)

[34] G. Di Sciascio, in CRIS 2015 proceedings, Preprint arXiv: 1602.07600

[35] Z. Cao et al., Chin. Phys. C 34, 249 (2010)

[36] P. Assis et al., preprint arXiv: 1607.03051 